\title{
Neonatal withdrawal from maternal volatile substance abuse
}

\author{
Milton Tenenbein, Oscar G Casiro, Mary M K Seshia, Val D Debooy
}

\begin{abstract}
Aim-To determine whether neonates born to mothers who are volatile substance abusers are at risk for an abstinence syndrome.

Methods-A consecutive sample of infants born to volatile substance abusing mothers was studied over four years, in a university affiliated medical centre with a variable mix of primary, secondary, and tertiary care patients. Infants were clinically scored with the Finnegan Neonatal Abstinence Scoring System. Those who fulfilled a priori scoring criteria were treated with phenobarbital and scoring was continued.

Results-There were 48 babies of whom 32 fulfilled the criteria for pharmacotherapy. All eight babies with the characteristic odour, and 15 of the 21 born to mothers with that odour, fulfilled these criteria. The typical symptoms were excessive and high pitched cry, sleeplessness, hyperactive Moro reflex, tremor, hypotonia, and poor feeding. Mean age of onset of treatment was $27 \cdot 1$ hours and mean duration was 5.8 days. Treatment was judged effective in 17 of 27 , while benefit was borderline in three and absent in seven. Conclusions-It is suggested that there is an identifiable neonatal volatile substance abuse abstinence syndrome. The characteristic chemical odour in the neonate or mother is a marker for its occurrence, and phenobarbital treatment seems to be effective. The Finnegan Scoring System seems to be useful for grading its severity. (Arch Dis Child 1996; 74: F204-F207)
\end{abstract}

Keywords: volatile substance abuse abstinence syndrome, substance abuse, neonatal withdrawal, maternal.

Volatile substance abuse (also known as inhalant abuse) occurs endemically throughout the world. ${ }^{1}$ Annual surveys in the United States consistently find that $15-20 \%$ of American high school seniors have abused volatile substances. ${ }^{2}$ In the United Kingdom 100 deaths are documented every year. ${ }^{3}$ The prevalence of chronic abuse has not been established. Recently, the American National Institute on Drug Abuse (NIDA) released the findings of its National Pregnancy and Health Survey. They estimated that 12000 infants every year are born to mothers who have abused volatile substances during pregnancy (Press Release, NIDA, September 12 1994). Although withdrawal has been described in adults, ${ }^{4}$ there is no report of a neonatal withdrawal syndrome. We describe an abstinence syndrome in a large cohort of neonates born to volatile substance abusing mothers, the grading of its severity using a clinical scoring system, and its treatment with phenobarbital.

\section{Methods}

All infants born in our university affiliated medical centre from 1 January 1989 to 31 December 1992 were eligible for study. The obstetric practice of this institution includes a variable mix of primary, secondary, and tertiary care patients. Inclusion criteria were infants of mothers with a history of volatile substance abuse during pregnancy, or in whom the obvious hydrocarbon odour was detected during admission for labour and delivery.

The history of volatile substance abuse was obtained in one of two ways: from a short questionnaire addressing general health and substance abuse history routinely administered to all patients admitted to the labour and delivery unit; or from a communication known as the 'birth alert'. This is a letter sent to all obstetric units in our city by social service agencies as soon as they are aware of a substance abusing pregnant woman. It is written by a social worker who has detailed case information, including the specific type of substance abuse. Exclusion criteria were neonates with major medical or surgical illness in whom signs and symptoms of withdrawal would probably be confounded by the primary condition. Neonates born to mothers who abused other illicit substances in addition to volatile substances were also excluded, but alcohol use was an exclusion criterion only if it was the primary or preferred substance of abuse.

The clinical scoring instrument used was the Finnegan Neonatal Abstinence Scoring System. ${ }^{5}$ It assigns a specific numeric score of 0 to 3 to each of 21 signs known to occur in passively addicted neonates. Higher scores are assigned based on the severity of the signs. The bedside nurse scores each infant at two hourly intervals and the total score for each interval is determined by summing the scores for the individual signs. The maximum score is 37 . All data were systematically collected on a standardised form. This scoring system is routinely used for all neonates at risk of withdrawal regardless of the substance of abuse, and its use had been implemented well before the start of the study period. Interobserver variability was not assessed.

Phenobarbital was used to treat withdrawal. Criteria for treatment were an average score of 8 or greater obtained on three consecutive two 
hour intervals, or 12 or greater on two consecutive intervals. A loading dose of $20 \mathrm{mg} / \mathrm{kg}$ was followed by a maintenance dose of 5 $\mathrm{mg} / \mathrm{kg} /$ day. Serum phenobarbital concentrations were not monitored and pharmacotherapy was not titrated against subsequent clinical scoring. Pharmacotherapy was maintained for a minimum of three days and was discontinued by the attending physician depending on the clinical score. There were no a priori criteria for this decision. Efficacy of pharmacotherapy was retrospectively determined as present, absent, or borderline by assessing its effect on the clinical scores. An improved score marks decreased neonatal distress, and improved handling and feeding.

Maternal and neonatal medical records were retrospectively reviewed for the presence of chemical odours characteristic of volatile substance abuse in the mothers or their newborns.

\section{Results}

There were 16160 deliveries during the study period and 60 neonates were identified as having in utero exposure to volatile substances. Twelve babies born to 11 mothers were excluded.

There was one twin pregnancy. Seven were excluded because they were critically ill; six of these were premature. The other five were excluded because the history of volatile substance abuse was not known at the time of delivery and thus these babies were not scored. Two of these neonates were premature.

There were 48 neonates delivered to 38 women who were included in the study. One woman delivered three of the studied newborns and eight others each delivered two. There were no multiple births. Thirty two of the neonates met the criteria for pharmacotherapy and their clinical features are contrasted with the other 16 in table 1 .

Notably, all eight newborns with the chemical odour met the criteria for treatment. Their mothers also had the odour, and several of these women were obviously impaired as a consequence of volatile substance abuse immediately before presentation to hospital. Fifteen of the 21 neonates born to mothers with the odour also fulfilled the criteria for treatment. Three of the remaining six approached these criteria.

Metabolic acidosis was frequently documented (table 1). It was present during the first 24 hours of life, and two different patterns were observed. The more common was transient, either spontaneously remitting or responding to a single dose of sodium bicarbonate. The other was persistent, lasting up to one to two weeks, and requiring treatment with repeated doses of sodium bicarbonate or Shohl's solution.

Nineteen of the newborns had siblings in the study. Five of these 19 did not fulfil the criteria for treatment, but all five had siblings who did. Birth order was random with respect to need for pharmacotherapy. In five siblings treatment was indicated for both members. (The sibship of three is found in both of these subcohorts).
Table 1 Clinical features of 48 neonates exposed to volatile substances in utero

\begin{tabular}{lcc}
\hline Fulfilled criteria for & & \\
pharmacotherapy & Yes & No \\
Number of newborns & 32 & 16 \\
Median birthweight (g) & 2885 & 2685 \\
Range & $2040-4080$ & $1750-4190$ \\
Sex: & 20 & 9 \\
Male & 12 & 7 \\
Female & 8 & 0 \\
Chemical odour: & 15 & 6 \\
Newborn & $17 / 22$ & $5 / 14$ \\
Mother & 11 & 4 \\
Metabolic acidosis & 6 & 1 \\
Transient & & \\
Persistent & & \\
\hline
\end{tabular}

^Blood gases were not measured in all neonates.

Thus the presence of, or the lack of a history of, neonatal volatile substance abuse abstinence syndrome in a sibling cannot be used to predict the likelihood for its subsequent occurrence.

The typical features of the abstinence syndrome were excessive and high pitched cry, sleeplessness, tremors, hypertonia and poor feeding. The other features scored in the Finnegan System - sweating, fever, yawning, mottling, nasal stuffiness, sneezing, nasal flaring, tachypnoea, excessive sucking, vomiting and diarrhoea - were typically absent. That two thirds of the cohort fulfilled the criteria for treatment when many of the criteria in the scoring system were not applicable, underscores the intensity of the neurological abnormality in these neonates.

As only 28 of the 32 newborns who met the criteria for pharmacotherapy were treated, there were four protocol violations. Additionally, one of the 28 was not given maintenance treatment, despite receiving a loading dose. Details and evaluation of pharmacotherapy are given in table 2 .

Notably, only seven of these 27 newborns did not show improvement.

The benefits of treatment were less crying and improved feeding and sleeping habits. Hospital stay was not shorter for those who were treated, because both groups of babies remained in hospital longer than medically indicated while awaiting foster home placement.

\section{Discussion}

Although volatile substance abuse occurs worldwide, its prevalence is unknown and it is not likely to be homogenously distributed. Our catchment area has a relatively high prevalence. One reason for this is a lower prevalence of other substances of abuse, such as cocaine and opiates, as documented by the results of drug screens done in our hospitals. Therefore,

Table 2 Details of newborn infants given treatment

\begin{tabular}{lc}
\hline Number of newborns & 28 \\
Age at onset of treatment & $27 \cdot 1$ hours \\
Duration of treatment $(n=27)^{\star}$ & $5 \cdot 8$ days \\
Efficacy of treatment $(n=27)^{\star}$ & 17 \\
Yes & 7 \\
No & 3 \\
Borderline &
\end{tabular}

*One newborn received a loading dose but no maintenance treatment. 
volatile substances are used as a substitute. This may explain why we were able to accumulate such a large cohort and document a phenomenon that had not been reported before.

Withdrawal from volatile substance abuse has been documented in adolescents and adults $^{4}$ and has been seen on numerous occasions by one of us (MT). These patients had engaged in prolonged binges. That the presence of a chemical odour in the newborn or mother was found to be a risk factor is not surprising because it is an obvious marker for recent abuse. The odour can persist for several days after delivery. This occurs because the lungs are a major route of excretion. Because these chemicals are highly lipophilic, prolonged periods of time are required for excretion, so that the odour can be detected on the baby's breath during that time.

The efficacy of pharmacotherapy was satisfactory given that there was no attempt to evaluate bioavailability with serum phenobarbital concentrations or to titrate dose against clinical scores. Had we done this, it is likely that the resultant treatment effect would have been stronger.

Metabolic acidosis as a complication of volatile substance abuse has been well documented in adults. ${ }^{67}$ The precise pathophysiology is uncertain with possible aetiologies being the acid load of toluene metabolites or renal tubular acidosis. Metabolic acidosis in babies born to volatile substance abusing mothers has been briefly documented. ${ }^{89}$

Descriptive data regarding volatile substance abuse practices in our cohort were not collected. These include age of onset, frequency of use, and types of volatile substances abused during pregnancy. In our experience responses to these questions are unreliable, which is a uniform feature of other types of substance abuse. The chief products abused by these women were solvents used by painters, lacquers, and glue. Spray paint and butane are virtually never used in our catchment area. Although toluene is found in all of the above products abused by these women, other organic hydrocarbons are also present. The heterogeneity of chemicals to which the abuser is exposed during volatile substance abuse is a common feature wherever this type of substance abuse occurs and it significantly confounds the study of this problem.

Alcohol use during pregnancy was not an exclusion criterion. Although withdrawal in neonates born to mothers who abuse alcohol has been described, the reports are surprisingly few. ${ }^{10-12}$ In all of these reports, the odour of alcohol was apparent during labour and some of these women were profoundly inebriated. ${ }^{11} 12$ Onset of withdrawal was typically within the first several hours of life in contrast to the later onset seen in our cohort. In two prospective studies that looked for symptoms and signs of neonatal alcohol withdrawal, the findings were not obvious, as they required sophisticated neurodevelopmental assessment for their demonstration. ${ }^{1314}$ Interestingly, Coles et al subsequently published a follow up study. ${ }^{15}$
They found that the abnormalities remained at 30 days of life, leading them to conclude that their findings at day 3 were the initial documentation of persistent neurodevelopmental abnormalities rather than withdrawal. ${ }^{15} 16$ For these reasons we do not believe that ethanol use was a confounding factor in our cohort.

Other issues that should be addressed include our study's descriptive rather than controlled design, the possibility of interrater variability during scoring, and a lack of neonatal urine drug screens. Studying both the scoring and the pharmacotherapy in a controlled manner would have been ideal. However, blinding the scorers is not possible for many reasons. The presence of the odour and the need for prominent display of the order indicating that guardianship has been assumed by the state are two features that would identify subjects as study rather than control babies. Studying the effectiveness of phenobarbital treatment in a placebo controlled blind manner would have been a stronger design. However, the observation that pharmacotherapy was not effective in all of our patients, although likely dose related, mitigates against observer bias.

Although interrater reliability for scoring was not assessed, it is important to note that this procedure was a routine practice in our newborn nursery which had been instituted long before the study period.

We did not perform neonatal drug screens. However, a positive urine drug screen only confirms recent exposure; therefore, it supports, but does not prove, that the infant is withdrawing from that specific substance. In fact, withdrawal can occur in infants with negative drug screens because it is the absence of a clinically relevant amount of the drug that is responsible for the abstinence syndrome. ${ }^{17}$ Neonatal heroin withdrawal has been documented in infants with negative toxicology screens. ${ }^{18}$

A history of heavy maternal use is more important. In adults with this use pattern, volatile substances are typically the only drugs that are abused. ${ }^{19}$ Besides preferring these over other substances of abuse, volatile substance abusers usually lack the social contacts and economic resources to obtain hard drugs such as amphetamines and heroin. Most of our patients are well known to our institution where they seek primary care. Many of them frequently visit the emergency department under the influence of volatile substances during which time other substance abuse was not detected. The social workers who write the 'birth alerts' also have intimate knowledge of their clients' substance abuse histories. Of additional importance is that the prevalence of abuse of other drugs associated with a neonatal abstinence syndrome, such as opiates and amphetamines, is low in our community relative to larger metropolitan areas. This is likely to be one of the reasons for our high rate of volatile substance abuse. Furthermore, the symptom complex in our group of neonates is different from that of other neonatal abstinence syndromes, most notably for a lack of 
gastrointestinal symptoms. These factors, along with the size of our cohort, make it quite unlikely that our babies were withdrawing from other substances of abuse.

We conclude that there is a neonatal volatile substance abuse abstinence syndrome for which the Finnegan Scoring System seems to be useful for the grading of its severity. A chemical odour characteristic of volatile substances in the neonate or mother is a marker for its occurrence, and phenobarbital treatment seems to be effective.

1 Kerner K. Current topics in inhalant abuse. In, Crider RA, Rouse BA, eds. Epidemiology of inhalant abuse: an update. NIDA Research Monograph 85. Washington, DC: US Department of Health and Human Services, 1988: 8-29.

2 Beauvais F. Volatile solvent abuse: trends and patterns. In, Sharp CW, Beauvais F, Spence R, eds. Inhalant abuse: a volatile research agenda. NIDA Research Monograph 129. Washington, DC: US Department of Health and Human Services 1992: 13-42.

3 Ramsey J, Anderson HR, Bloor K, Flanagan RJ. An introduction to the practice, prevalence and chemical toxicology of volatile substance abuse. Hum Toxicol 1989; 8 261-9.

4 Evans AC, Raistrick D. Phenomenology of intoxication with toluene-based adhesives and butane gas. $\mathrm{Br} \mathcal{F}$ Psychiatry 1987; 150: 769-73.

5 Finnegan LP, Kron RE, Connaughton JF Jr, Emlich JP Jr. A scoring system for evaluation and treatment of the neonatal abstinence syndrome: a new clinical and research tool. In: Morselli PL, Garattini S, Sereni F, eds. Basic and tool. In: Morselli PL, Garattini S, Sereni F, eds. Basic and
therapeutic aspects of perinatal pharmacology. New York:
Raven Press, 1975: 139-53.

6 Taher SM, Anderson RJ, McCartney R, Popovtzer MM,
Schrier RW. Renal tubular acidosis associated with toluene sniffing. N Engl f Med 1974; 290: 765-8.

7 Fischman CM, Oster JR. Toxic effects of toluene. A new cause of high anion gap metabolic acidosis. f $A M A 1979$ 241: 1713-15.

8 Goodwin TM. Toluene abuse and renal tubular acidosis in pregnancy. Obstet Gynecol 1988; 71: 715-18.

9 Wilkins-Haug L, Gabow PA. Toluene abuse during pregnancy. Obstetric complications and perinatal outcomes. Obstet Gynecol 1991; 77: 504-9.

10 Pierog S, Chandavasu O, Wexler I. Withdrawal symptoms in infants with fetal alcohol syndrome. $\mathcal{F}$ Pediatr 1977; 90: 630-3.

11 Schaefer O. Alcohol withdrawal syndrome in a newborn infant of a Yukon Indian mother. Can Med Assoc f 1962 87: 1333-4.

12 Nichols MM. Acute alcohol withdrawal syndrome in a newborn. Am f Dis Child 1967; 113: 714-15.

13 Coles CC, Smith IE, Fernhoff PM, Falek A. Neonatal ethanol withdrawal: characteristics in clinically normal

( and neonatal habituation assessed with the Brazelton Scale. Child Devel 1983; 54: 1109-118.

15 Coles CD, Smith IE, Lancaster JS, Falek A. Persistence over the first month of neurobehavioral differences in infants exposed to alcohol prenatally. Infant Behavior and infants exposed to alcohol prena

16 Coles CD, Smith IE, Falek A. Prenatal alcohol exposure and infant behaviour: immediate effects and implications for later development. Adv Alcohol Subst Abuse 1987; 6: 87-104.

17 Desmond MM, Wilson GS. Neonatal abstinence syndrome. Recognition and diagnosis. Addictive Diseases 1975; 2: 113-21.

18 Fulroth R, Phillips B, Durand DJ. Perinatal outcome of infants exposed to cocaine and/or heroin in utero. $\mathrm{Am} \mathcal{F}$ Dis Child 1989; 143: 905-10.

19 Oetting ER, Edwards RW, Beauvais F. Social and psychological factors underlying inhalant abuse. In: Crider RA, Rouse BA, eds. Epidemiology of inhalant abuse: an update. NIDA Research Monograph 85. Washington, DC: US Department of Health and Human Services, 1988: 172-203. 